\title{
Structural analysis of a tetradecameric HSP100 chaperone by cryo-EM and SAXS
}

\author{
Hyunwoo Cho, Gyuhee Kim, Sangho Lee
}

SungKyunKwan University, Suwon, Korea, Republic of (South Korea);

gusdnf0326@naver.com

Heat shock protein 100 (HSP100) family members serve as ATPases associated with diverse cellular activities (AAA+) chaperones. HSP100s possess three enzymatic activities: ATPase, refoldase and disaggregase/holdase. With these activities, HSP100s play key roles in cellular stress responses and protein homeostasis. The functional oligomeric state of HSP100 is mostly modulated by their hexameric/dodecameric quaternary structures as exemplified by studies on $\mathrm{ClpB}$ and $\mathrm{ClpC}$. However, little is known about nonhexameric/dodecameric HSP100 chaperones. We found that ClpL from Streptococcus pneumoniae forms tetradecamers in solution. Here we report the structural characterizations of the tetradecameric $\mathrm{ClpL}$ to reveal key residues responsible for the tetradecameric assembly. Cryo-EM structure of ClpL reveals a striking tetradecameric arrangement where two heptameric rings are connected by vertical middle domains. Non-conserved residues, Q321 and R670, are crucial in the heptameric ring assembly of ClpL while hydrophobic F350 contributes to the interface among the middle domains. Site-directed mutagenesis analysis supported the differential roles of the aforementioned residues. Mutations in Q321 and R670 abrogated ATPase and refolase activities, supporting that these residues are critical in the integrity of the heptameric ring arrangement. Mutations in hydrophobic residues of the middle domain deteriorated refoldase and disaggregase/holdase activities. Solution structures of ClpL derived from small-angle X-ray scattering (SAXS) data suggest that the tetradecameric ClpL could assume a spiral conformation found in active hexameric/dodecameric HSP100 chaperone structures. These results establish that ClpL is a functionally active tetradecamer, clearly distinct from hexameric/dodecameric HSP100 chaperones.

Keywords: Streptococcus pneumoniae, Chaperone, HSP100, AAA+ ATPase, Tetradecamer 